\title{
Microscopie X analytique et diffusion des ions en solution
}

\author{
S. Rondot et J. Cazaux \\ L.A.S.S.I., UFR Sciences Exactes et Naturelles, BP. 1039, 51687 Reims cedex 2, France
}

\begin{abstract}
Résumé : Un microscope à rayons $\mathrm{X}$ basé sur la simple modification d'un microscope électronique à balayage permet, grâce à l'emploi d'une caméra CCD, de suivre in situ, au cours du temps avec une résolution latérale de l'ordre de $10 . \mu \mathrm{m}$ le processus de diffusion d'une seule espèce ionique en solution. Une radiation suffit alors pour déterminer la concentration locale des ions en solution. Au moyen d'un formalisme mis au point au laboratoire, il est possible d'obtenir les cartographies élémentaires des n constituants d'un échantillon et la cartographie des épaisseurs moyennant l'utilisation de $n$ radiations $\mathrm{X}$. Cette méthodologie a permis de quantifier la diffusion de deux espèces ioniques dans un liquide au cours d'une réaction chimique classique.
\end{abstract}

\begin{abstract}
An X-ray microscope, implemented from a simple modification of a Scanning Electron Microscope, permits to follow, with the help of a CCD camera, the diffusion process of one ionic species in an aqueous solution, as a function of time and with a lateral resolution on the order of $10 \mu \mathrm{m}$. Quantitative analysis of this species requires only one $X$-ray irradiation. Using a new formalism one can obtain $\mathbf{n}$ elemental maps and thickness map of a specimen using $\mathbf{n}$ X-ray irradiations. Our method has been applied to follow quantitatively two ionic species diffusing in a liquid during a standard chemical reaction.
\end{abstract}

\section{INTRODUCTION}

La microscopie $X$ par projection exploite l'aptitude des rayons $X$ à traverser des échantillons épais éventuellement placés dans un environnement choisi ainsi que leur sensibilité dans la détection d'éléments moyens ou lourds $(\mathrm{Fe}, \mathrm{Au})$ insérés dans une matrice légère $\left(\mathrm{C}, \mathrm{H}_{2} \mathrm{O}\right)$.

Elle permet donc l'analyse quantitative des liquides, surtout s'agissant des solutions couramment utilisées en chimie et en électrochimie, qui font intervenir des ions métalliques dans un milieu constitué essentiellement d'eau. L'observation dynamique de la diffusion des ions constitue un nouveau domaine dintérêt pour la microscopie $\mathrm{X}$. Cette approche déjà initiée par P. Anderson et al [1] et $\mathbf{S}$. Rothman et al [2], a été améliorée au laboratoire par la possibilité de visualiser directement le phénomène de diffusion par l'acquisition d'une série de microradiographies du processus au cours de son déroulement. Il a été ainsi possible de suivre directement la diffusion d'ions zinc dans une solution d'acide chlorhydrique résultant de la corrosion d'une électrode de zinc pur [3]. L'intérêt de ce type d'expérience est renforcé par la possibilité d'évaluer la concentration locale des ions dans la solution et leur évolution au cours du temps. Lorsque plusieurs constituants interviennent dans le processus, un formalisme approprié [4] permettant d'obtenir les cartographies quantitatives des espèces a été mis en oeuvre et sa validité vérifiée sur un échantillon test solide [5]. La combinaison de cette méthodologie avec les études dynamiques de processus de diffusion permet d'analyser des solutions plus complexes dans lesquelles plusieurs espèces interagissent. Ce qui ouvre le champ à de nombreuses applications dans le domaine de la chimie et de l'électrochimie. 


\section{LE MICROSCOPE X DU LABORATOIRE}

L'instrument mis au point au laboratoire [6], [7] est schématisé sur la figure 1. Il s'agit d'un microscope $\mathrm{X}$ par projection dérivé de l'architecture d'un microscope électronique à balayage (MEB). La source $X$ est obtenue par bombardement électronique d'une anticathode sous forme d'un film mince (quelque $\mu \mathrm{m}$ d'épaisseur). Le faisceau conique de rayons $\mathrm{X}$ ainsi généré conduit à l'obtention sur un phosphore convertisseur d'une image d'absorption de l'objet situé entre la source $X$ et le convertisseur. Par rapport au M.E.B. initial, les seules modifications concernent d'une part l'adjonction d'un porte anticathode mobile permettant la substitution d'une cible à l'autre par translation (et donc le changement de l'énergie des photons $X$ caractéristiques émis), d'autre part la mise en place d'une caméra CCD refroidie, précédée de l'écran convertisseur, comme détecteur $2 \mathrm{D}$ de rayons $\mathrm{X}$. La résolution latérale du microscope $X$ est donnée par l'expression $r_{g}=s+\frac{D-s}{G}$ où $s$ et $D$ sont respectivement la taille de la source $\mathrm{X}$ et la taille d'un détecteur élémentaire. $\mathrm{G}$ est le grandissement obéissant à la relation $G=\frac{a+b}{a}$.

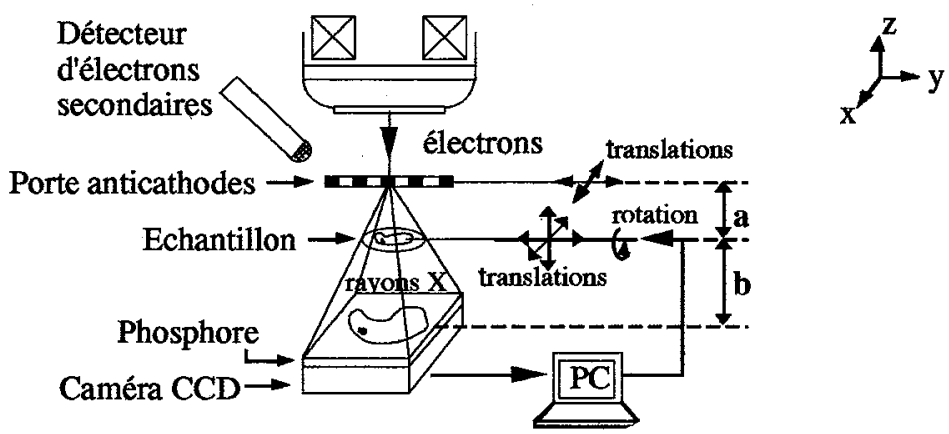

Figure 1: Schéma du microscope $\mathrm{X}$ du laboratoire.

Diagrammatic of our X-ray microscope.

Signalons que ce même instrument permet la reconstruction tridimensionnelle des objets biologiques (microtomographie $X$ [8]), et même l'imagerie par réflexion totale des rayons $X$ et la diffraction à incidence rasante en faisceau divergent. [9]

\section{ETUde DYNAMIQUE DE LA DIFFUSION D'UN SEUL TYPE D'ION EN SOLUTION}

Grâce au détecteur $\mathrm{CCD}$, la rapidité d'acquisition des images numériques permet de suivre in situ et au cours du temps la diffusion d'une espèce ionique en solution aqueuse résultant d'une réaction chimique ou électrochimique. La microscopie $\mathrm{X}$ permet donc de visualiser et de quantifier directement les ions en mouvement dans une solution à l'échelle de quelques $\mu \mathrm{m}$ contrairement aux mesures globales de l'électrochimie ou aux approches purement théoriques (et numériques) relatives à la résolution des équations de Fick. L'échantillon étant dans le vide de la chambre objet, le suivi de ces phénomènes en solution a nécessité la conception d'une cellule étanche en matériau léger (PTFE, polypropylène).

Les résultats obtenus concernent l'étude de la corrosion dissolution du zinc [3] dont le bilan se résume par l'équation : $\mathrm{Zn}+2 \mathrm{H}^{+} \rightarrow \mathrm{Zn}^{2+}+\mathrm{H}_{2}$ 
Une série de microradiographies acquises pendant le déroulement du processus permet de visualiser directement les ions $\mathrm{Zn}^{2+}$ en mouvement dans la solution. (Fig. 2).

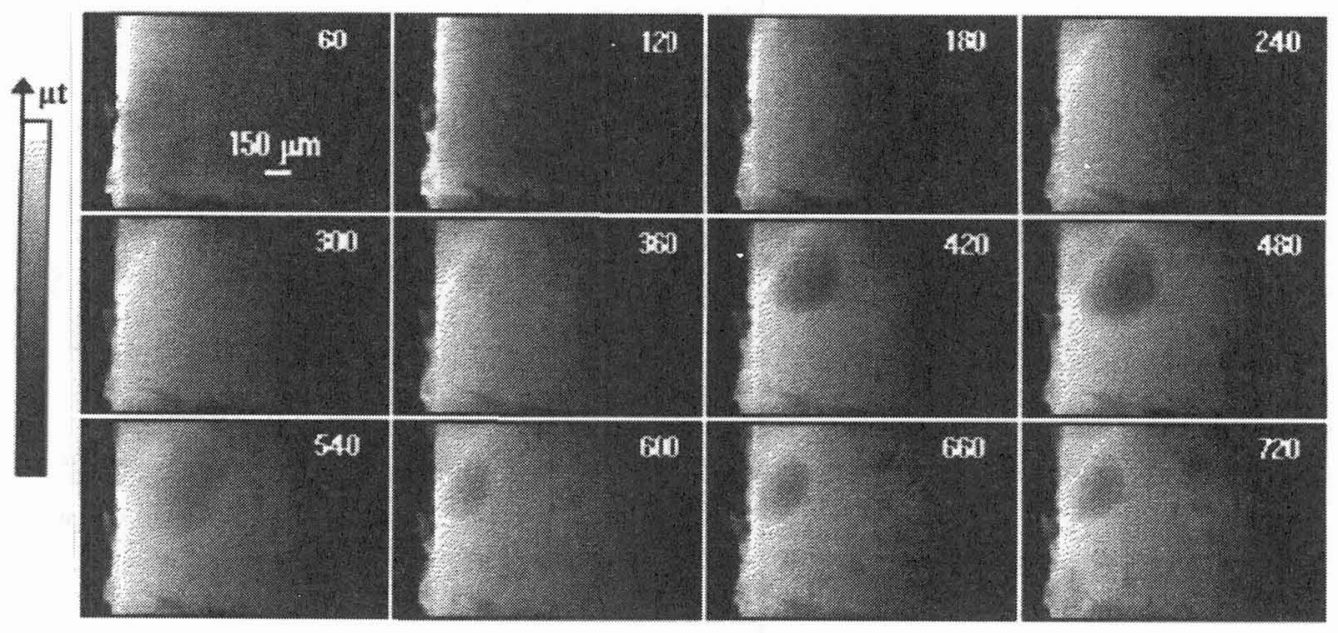

Figure 2 : Suivi de la diffusion des ions zinc (en gris clair au centre de chaque image). On distingue, à gauche, l'électrode de zinc sur laquelle les zones noires correspondent à la perte de matière. L'image de la cellule ne contenant que l'électrode a été soustraite pixel par pixel à toutes les images acquises toutes les mimutes (temps en secondes en haut à droite des images) pendant la réaction (Conditions expérimentales: anticathode Mo ; Eo = $29 \mathrm{keV} ; \mathrm{Io}=0,48 \mu \mathrm{A}$ ).

Corrosion of a zinc plane electrode (in black on the left of each image) set in contact with a hydrochloric acid solution. Black area on electrode are corrosion pits. Microradiographs were obtained by substracting pixel by pixel the initial $\mu t$ image (acquired at $t=0$ before any chemical reaction) from the successive $\mu t$ images (acquired at the indicated time in seconds during the chemical reaction). Light area correspond to the strong $X$ ray absorption of the $\mathrm{Zn}^{2+}$ ions relative to that of the PTFE and dilute acid. Dark regions characterize weaker absorption (Experimental condition: Target $\mathrm{Mo} ; \mathrm{Eo}=29 \mathrm{keV}$; $\mathrm{Io}=0,48 \mu \mathrm{A}$ ).

Les images acquises sous forme numérique se prêtent directement à des mesures quantitatives par compression logarithmique des images successives puis soustraction de ces images de l'image initiale ce qui conduit à une cartographie de $\mu \mathrm{t} s$ 'affranchissant de la contribution de la cellule. La figure 3 illustre la possibilité de déterminer, pour chaque pixel des images, l'évolution de la concentration des ions zinc dans la solution en fonction du temps.

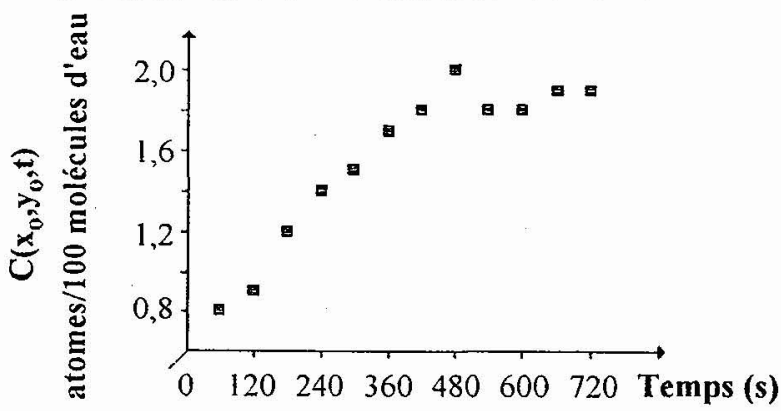

Figure 3 : Evolution de la concentration des ions zinc pour un pixel donné des microradiographies Evolution of the zinc ions concentration for a given pixel of the images.

Dernièrement, il a été aussi possible d'étudier les variations de concentration des ions cuivre en solution au cours d'un dépôt de cuivre sur une électrode de carbone. Les dendrites de cuivre 
formées sur l'électrode engendrent de fortes variations locales de la concentration des ions en leur voisinage (figure 4). La microscopie $X$ numérique permet donc aussi l'analyse quantitative locale des variations de concentration des ions cuivre ainsi que leur évolution en fonction du temps par acquisition d'une série de microradiographies.
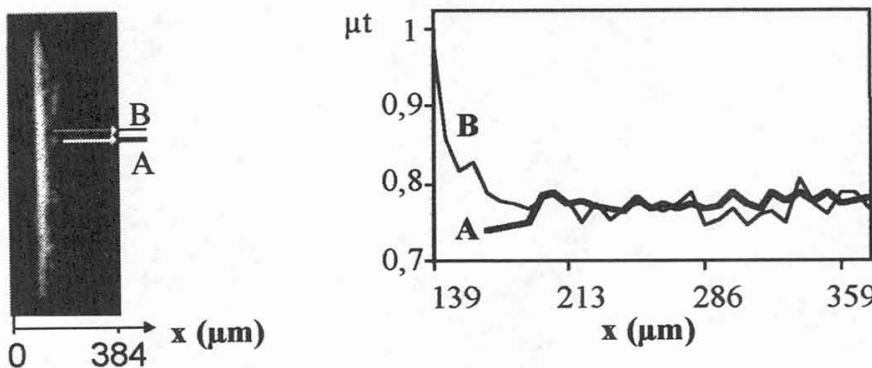

Figure 4 : Electrodéposition du cuivre sur carbone vitreux. A gauche : une image du processus parmi la série acquise . A droite profils de concentration du cuivre en solution le long des lignes A et B [5].

Electrodeposition of copper on a glassy carbon electrode. Left: an image of the process from the time series of microradiographs acquired. Righ: Concentration profile of copper in solution along the lines $A$ and $B$.

\section{MICROSCOPIE X ANALYTIQUE}

Les études précédentes ont été effectuées à l'aide d'une seule radiation $\mathrm{X}$ caractéristique pour quantifier la distribution d'une espèce en solution. Lorsque $n$ espèces interviennent dans un milieu réactionnel, $\mathrm{n}$ radiations $\mathrm{X}$ et un formalisme approprié sont nécessaires pour les visualiser. Deux nouvelles méthodologies pour l'analyse quantitative en microscopie $\mathrm{X}$ ont été proposées au laboratoire [4] [10]. La première a fait l'objet d'une vérification expérimentale concluante [5]. Elles sont basées sur le fait que le coefficient d'absorption linéaire $\mu\left(E_{1}\right)$, pour une énergie $E_{1}$ des photons $X$, obéit à la relation :

$$
\begin{aligned}
\mathrm{Fe}+\mathrm{Cu}^{2+} & \rightarrow \mathrm{Fe}^{2+}+\mathrm{Cu} \\
\mu\left(\mathrm{E}_{1}\right) \mathrm{t} & =\mathrm{N}_{0} \mathrm{t} .\left(\mathrm{C}_{\mathrm{A}} \sigma_{\mathrm{A}}\left(\mathrm{E}_{1}\right)+\mathrm{C}_{\mathrm{B}} \sigma_{\mathrm{B}}\left(\mathrm{E}_{1}\right)+\ldots+\mathrm{C}_{\mathrm{N}} \sigma_{N}\left(\mathrm{E}_{1}\right)\right)
\end{aligned}
$$

dans laquelle $C_{N}$ et $\sigma_{N}\left(E_{1}\right)$ représentent la concentration atomique et la section efficace d'absorption de l'élément $\mathrm{N}$, et $\mathrm{N}_{\mathrm{o}} \mathrm{t}$ la densité superficielle $\left(\mathrm{at} / \mathrm{cm}^{2}\right)$ locale de l'échantillon (dans la zone associée à un pixel donné). L'usage de $n$ radiations $X$ d'énergies $E_{1}, E_{2} \ldots E_{n}$ conduit, après compression logarithmique des images obtenues, à $\mathrm{n}$ images donnant la répartition latérale du produit $\mu$ t et permet d'établir un système d'équations similaires à l'équation (1). Les concentrations sont alors déduites après une simple inversion de matrice pixel par pixel et la dernière inconnue $\mathrm{N}_{\mathrm{o}} \mathrm{t}$ est évaluée à partir de la règle de somme :

$$
\mathrm{C}_{\mathrm{A}}+\mathrm{C}_{\mathrm{B}}+\ldots+\mathrm{C}_{\mathrm{N}}=100 \%
$$

$\mathrm{Si} \mathrm{N}_{\mathrm{o}} \mathrm{t}$ est connu, le nombre de radiations nécessaires se réduit à $\mathrm{n}-1$ puisque la $\mathrm{n}^{\text {ième }}$ concentration se déduit de la relation (2).

La détermination des concentrations est effectuée très rapidement et la non monochromaticité du faisceau de rayons $\mathrm{X}$ partiellement corrigée à l'aide d'une procédure itérative. La figure 5 illustre la méthode appliquée à l'analyse quantitative d'un alliage ternaire. 

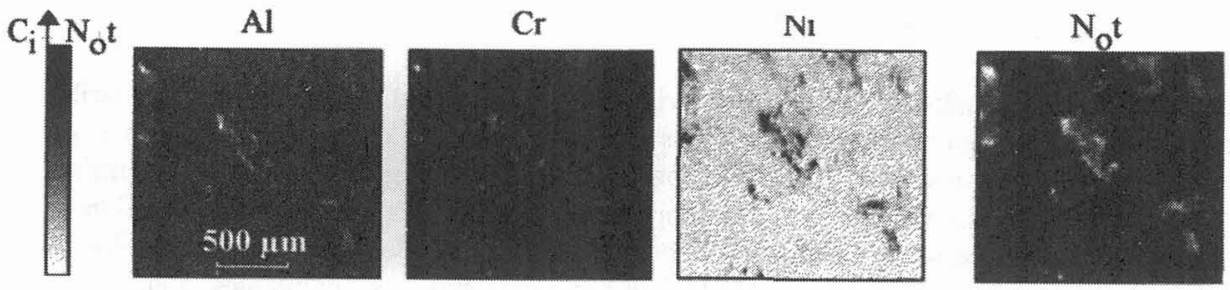

Figure 5 : Cartographies élémentaires d'un alliage métallique ternaire $\mathrm{Al}, \mathrm{Cr}, \mathrm{Ni}$ et cartographie des épaisseurs déduites des trois images acquises en utilisant les cibles de vanadium de Fer et de Zinc.

Elemental mapping of a ternary alloy $\mathrm{Al}, \mathrm{Cr}, \mathrm{Ni}$ and thickness map deduced from the three initial images acquired using $\mathrm{V}, \mathrm{Fe}$ and $\mathrm{Zn}$ targets.

\section{APPLICATION A LA CARTOGRAPHIE QUANTITATIVE DE DEUX ESPECES IONIQUES EN SOLUTION}

La combinaison étude dynamique + microscopie $X$ analytique a été réalisée pour l'étude de la dissolution d'une électrode de fer dans une solution de sulfate de cuivre. La réaction d'oxydoréduction se résume par le bilan : $\mathrm{Fe}+\mathrm{Cu}^{2+} \rightarrow \mathrm{Fe}^{2+}+\mathrm{Cu}$

Elle fait intervenir les composés $\mathrm{FeSO}_{4}, \mathrm{CuSO}_{4}$ et l'eau. Elle est mise en oeuvre dans une cellule étanche. L'épaisseur du liquide étant connue (hauteur de la cavité interne de la cellule) et considérée constante dans la zone irradiée, deux radiations $\mathrm{X}$ suffisent pour visualiser la distribution des composés $\mathrm{FeSO}_{4}$ et $\mathrm{CuSO}_{4}$.

L'acquisition de couples d'images de la solution à l'aide des deux radiations (FeK $\alpha$ et $\mathrm{NiK} \alpha$ ) à différents instants permet de suivre l'évolution de la distribution des deux composés au cours du temps après application du formalisme présenté précédemment.

La figure 6 représente une des cartographies établie à un instant donné de la réaction chimique $(t=4 \mathrm{mn})$ et les profils moyens de concentration des deux espèces. On notera l'inversion de ces profils au cours du temps ainsi que la sensibilité de la méthode proche de $10^{-5}$ molécule par molécule d'eau.
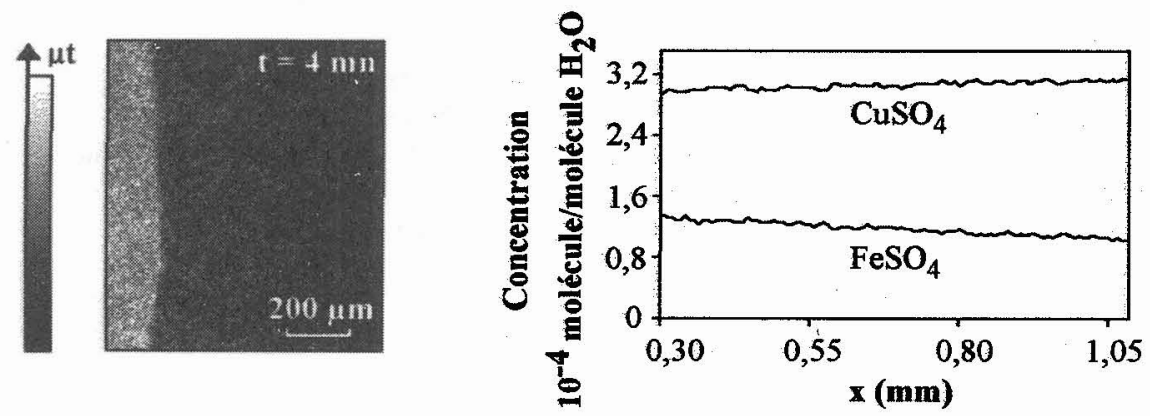

Figure 6 : A gauche : cartographie quantitative de $\mathrm{FeSO}_{4}, 4 \mathrm{mn}$ après le début du processus de dissolution/dépôt (la zone grise à gauche correspond à l'électrode de fer). A droite : profils de concentration moyens de $\mathrm{FeSO}_{4}$ et $\mathrm{CuSO}_{4}$ dans le liquide.

Left: Quantitative map of $\mathrm{FeSO}_{4}, 4 \mathrm{mn}$ after the beginning of the dissolution/deposition process (left grey area is the iron electrode). Right: mean concentration profiles of $\mathrm{FeSO}_{4}$, and $\mathrm{CuSO}_{4}$, in the solution. 


\section{CONCLUSION ET PERSPECTIVES}

Le présent article illustre la possibilité de visualiser directement l'évolution de la distribution de plusieurs espèces au cours d'un processus évoluant au cours du temps par microscopie $X$. Les études dynamiques sont valorisées par l'énorme avantage de pouvoir quantifier les différentes espèces en mouvement lent dans une solution. La microscopie $X$ quantitative constitue donc une méthode pertinente et inédite pour l'étude des mouvements des ions dans les liquides surtout lorsqu'on sait qu'il n'existe pas de techniques semblables pour quantifier aussi directement la distribution des espèces avec une résolution latérale d'une dizaine de $\mu \mathrm{m}$, la plupart des méthodes d'investigation à caractère analytique étant restreintes à l'analyse des solides.

Dans un proche avenir, il devrait être possible d'étudier les mouvements des ions (à l'échelle du $\mu \mathrm{m}$ ) au cours d'un processus électrochimique perturbé par une excitation thermique ou magnétique locale. Par ailleurs, nous envisageons de combiner dans une même expérience, étude dynamique, microscopie $X$ quantitative et microtomographie $X$ pour obtenir les cartographies $3 D$ des différentes espèces diffusant dans un solide ou un gel.

En regard de ces nouvelles applications et de ces perspectives, on ne peut douter de l'avenir de la microscopie $\mathrm{X}$ numérique qui devrait encore évoluer par l'avènement des caméras $\mathrm{CCD}$ analytiques en cours de développement [11].

\section{Références}

[1] Anderson P. et al, "X-Ray Microscoy III" Springer Series in Optical Sciences 67 (1992) 435-437

[2] Rothman S.S. et al in "X-Ray Microscoy III" Springer Series in Optical Sciences 67 (1992) 373-383

[3] Rondot S. et al, Science 263 (1994) 1739-1741.

[4] Cazaux J. Microscopy, Microanalysis, Microstructures, 4 (1993) 513-537.

[5] Rondot $S$., Thèse Université de Reims, à soutenir.

[6] Thomas X. et al, "X-Ray Microscoy III" Springer Series in Optical Sciences 67 (1992) 373-383

[7] Cazaux J. et al, Microscopy and Analysis 29 (1994) $19-21$

[8] Elhila H., et al, "Microscopie X et Microtomographie X: Application à la biologie " ce volume

[9] Erre D. et al, “ Prémices d'une imagerie 2D de surfaces et interfaces par réflexion totale des rayons $X$. Diffraction en incidence rasante" ce volume.

[10] Trebbia $P$. et al, "Quantitative elemental mapping by X-ray radiography: methods and programs " soumis à Microbeam Analysis (1995).

[11] Janesick J. et al, Microbeam Analysis (1995) ed. ES. Etz (Weinheim VCH) 7. 